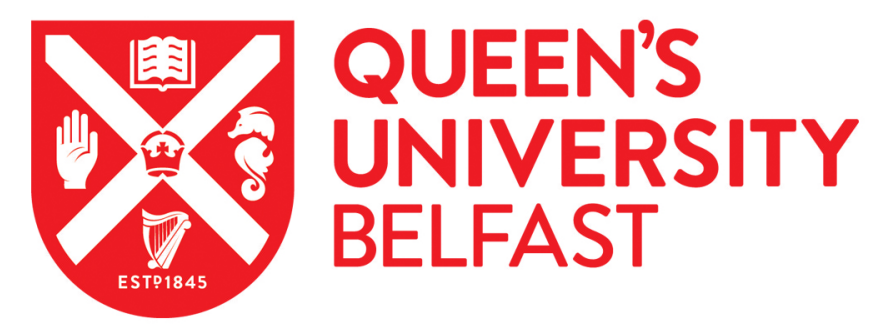

\title{
Avoidable Waste in Ophthalmic Epidemiology: A Review of Blindness Prevalence Surveys in Low and Middle Income Countries 2000-2014
}

Ramke, J., Kuper, H., Limburg, H., Kinloch, J., Zhu, W., Lansingh, V. C., Congdon, N., Foster, A., \& Gilbert, C. E. (2017). Avoidable Waste in Ophthalmic Epidemiology: A Review of Blindness Prevalence Surveys in Low and Middle Income Countries 2000-2014. Ophthalmic Epidemiology, 1-8.

https://doi.org/10.1080/09286586.2017.1328067

Published in:

Ophthalmic Epidemiology

Document Version:

Early version, also known as pre-print

Queen's University Belfast - Research Portal:

Link to publication record in Queen's University Belfast Research Portal

Publisher rights

Copyright 2017 Taylor and Francis. This work is made available online in accordance with the publisher's policies. Please refer to any applicable terms of use of the publisher.

\section{General rights}

Copyright for the publications made accessible via the Queen's University Belfast Research Portal is retained by the author(s) and / or other copyright owners and it is a condition of accessing these publications that users recognise and abide by the legal requirements associated with these rights.

Take down policy

The Research Portal is Queen's institutional repository that provides access to Queen's research output. Every effort has been made to ensure that content in the Research Portal does not infringe any person's rights, or applicable UK laws. If you discover content in the Research Portal that you believe breaches copyright or violates any law, please contact openaccess@qub.ac.uk. 


\title{
Avoidable waste in ophthalmic epidemiology: a review of blindness prevalence surveys in low and middle income countries 2000-2014
}

\author{
Jacqueline Ramke ${ }^{1}$ \\ Hannah Kuper ${ }^{2}$ \\ Hans Limburg ${ }^{3}$ \\ Jennifer Kinloch ${ }^{4}$ \\ Wenhui Zhu ${ }^{5}$ \\ Van C Lansingh ${ }^{6}$ \\ Nathan Congdon 789 \\ Allen Foster ${ }^{2}$ \\ Clare E Gilbert ${ }^{2}$
}

1. University of Auckland, School of Population Health, Faculty of Medicine and Health Sciences, Auckland, New Zealand

2. London School of Hygiene and Tropical Medicine, International Centre for Eye Health, Clinical Research Unit,

Department of Infectious \& Tropical Diseases, London, UK

3. Health Information Services, Nijenburg 32, 1613LC Grootebroek, Netherlands

4. Bath Spa University, College of Liberal Arts, Bath, UK

5. The First Affiliated Hospital of Sun Yat-sen University, Department of Ophthalmology, Guangzhou, Guangdong, China

6. Help Me See Latin America, Instituto Mexicano de Oftalmologia, Queretaro, Mexico

7. Queen's University Belfast, TREE, Centre for Public Health, Belfast UK

8. Sun Yat-sen University, Preventive Ophthalmology Department, Zhongshan Ophthalmic Center, Guangzhou, China

9. Orbis International, NY, USA

Corresponding Author: Jacqueline Ramke jramke@gmail.com

Running head: Avoidable waste in ophthalmic epidemiology

Financial support: None

Proprietary interests/ conflict of interest: No author has a conflict of interest to disclose. Authors were involved in the development of RAAB (HK and $\mathrm{HL})$ and the RAAB Repository $(\mathrm{HL})$, but do not benefit from their use.

Submission: This submission has not been published anywhere previously and it is not simultaneously being considered for any other publication. 


\section{Abstract \\ Purpose}

Sources of avoidable waste in ophthalmic epidemiology include duplication of effort, and survey reports remaining unpublished, gaining publication after a long delay, or being incomplete or of poor quality. The aim of this review was to assess these sources of avoidable waste by examining blindness prevalence surveys undertaken in low and middle income countries (LMICs) between 2000 and 2014.

\section{Methods}

On December 12016 we searched MEDLINE, EMBASE and Web of Science databases for crosssectional blindness prevalence surveys undertaken in LMICs between 2000-2014. All surveys listed on the Rapid Assessment of Avoidable Blindness (RAAB) Repository website ("the Repository") were also considered. For each survey we assessed i) availability of scientific publication, survey report, summary results tables and/or datasets; ii) time to publication from year of survey completion and journal attributes; iii) extent of blindness information reported; and iv) rigour when information was available from two sources (i.e. whether it matched).

\section{Results}

Of the 279 included surveys (from 68 countries) 186 (67\%) used RAAB methodology; 146 (52\%) were published in a scientific journal, 57 (20\%) were published in a journal and on the Repository, and $76(27 \%)$ were on the Repository only ( $8 \%$ had tables; $19 \%$ had no information available beyond registration). Datasets were available for 50 RAABs (18\% of included surveys). Time to publication ranged from $<1-11$ years (mean, standard deviation $2.8 \pm 1.8$ years). The extent of blindness information reported within studies varied (e.g. presenting and best-corrected, unilateral and bilateral); those with both a published report and Repository tables were most complete. For surveys published and with RAAB tables available, discrepancies were found in reporting of participant numbers (14\% of studies) and blindness prevalence (15\%).

\section{Conclusion}

Strategies are needed to improve the availability, consistency and quality of information reported from blindness prevalence surveys, and hence reduce avoidable waste. 


\section{Introduction}

Good quality evidence is required to achieve the ambitious goal of universal eye health set by the World Health Assembly in 2013. ${ }^{1}$ This evidence includes data from prevalence surveys on the magnitude and causes of visual impairment and blindness. Over the past two decades an increasing number of prevalence surveys have been undertaken, especially in low and middle income countries (LMICs). The current global eye health action plan of the World Health Organization (WHO) calls for more prevalence surveys to be undertaken to provide up-to-date local evidence for planning. ${ }^{2}$

An important factor in the recent increase in blindness prevalence surveys is the development of a protocol and software that can be downloaded freely from the internet ${ }^{3}$ - the Rapid Assessment of Avoidable Blindness (RAAB) and its predecessor the Rapid Assessment of Cataract Surgical Services (RACSS, hereafter collectively referred to as RAAB). RAAB is a population based survey of visual impairment in people aged 50 years and above that correlated well with data from total population surveys. ${ }^{4}$ Because RAAB surveys are restricted to those aged 50 years and above, where blindness prevalence is highest, ${ }^{4}$ the sample size is smaller, and the survey is shorter and less expensive than traditional surveys. ${ }^{5}$ RAAB uses simple examination methods, and the software includes a data entry and automated analysis package.

A consequence of the increasing number of blindness prevalence surveys is a reluctance by editors of scientific journals to publish them, as they offer little novelty in terms of purpose or methodology. ${ }^{6}$ In this publishing environment the online RAAB Repository ${ }^{7}$ (hereafter referred to as the Repository) has played an important role in dissemination of survey findings. The Repository was launched in May 2014 and by December 12016 the webmaster $(\mathrm{HL})$ had registered 266 RAABs on the Repository.

In 2009 Chalmers and Glasziou highlighted the issue of "avoidable waste" in the production and reporting of health research. ${ }^{8}$ In the context of ophthalmic epidemiology, sources of avoidable waste include duplication of effort (i.e. numerous surveys in the same area); and survey reports remaining unpublished, gaining publication after a long delay, or being incomplete or of poor quality. The aim of this review was to assess these sources of avoidable waste by examining blindness prevalence surveys undertaken in LMICs between 2000 and 2014. 


\section{Methods}

\section{Search strategy}

We sought to identify cross-sectional surveys of visual impairment and/or blindness undertaken in LMICs between the years 2000 and 2014. On December 12016 MEDLINE, EMBASE and Web of Science (2000 to November 2016) were searched without language restrictions using the algorithm 'blindness or vis* impairment or low vision' and 'prevalence or rapid assessment or populationbased'. To identify additional surveys to include, we examined reference lists of all articles selected for screening, as well as review articles of blindness prevalence. ${ }^{9-16}$ All surveys listed on the RAAB Repository website ${ }^{7}$ on December 12016 were reviewed.

\section{Study selection}

The titles of all citations identified during the initial search were systematically screened by two authors (JR and JK) to exclude publications that clearly did not meet the inclusion criteria. The fulltext article was retrieved for review if the citation was potentially relevant. For each eligible survey on the RAAB Repository all available reports, tables and datasets were downloaded.

The inclusion and exclusion criteria are outlined in Box 1.

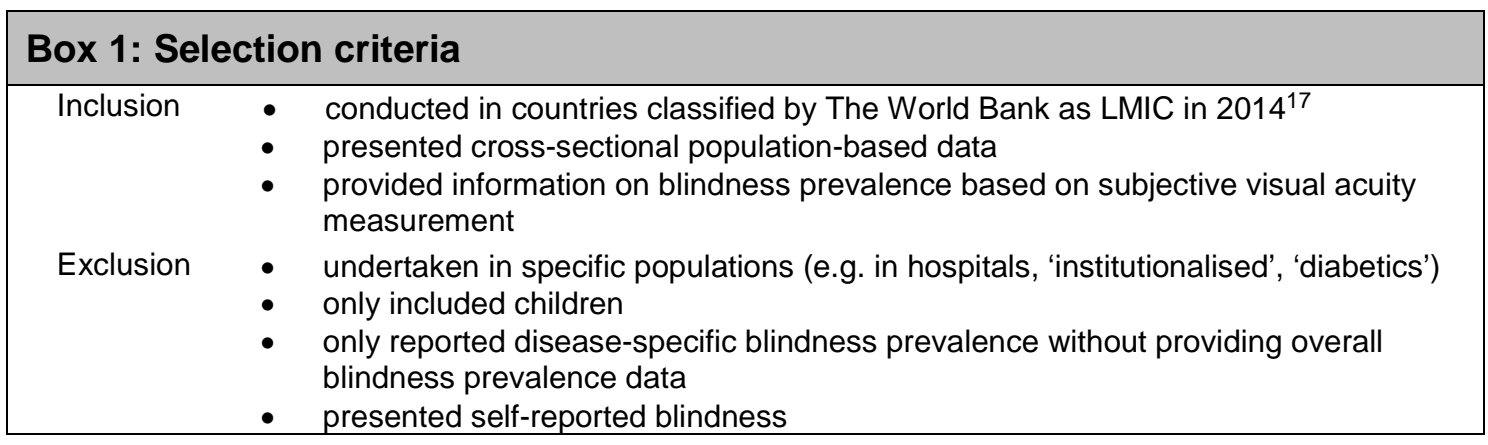

\section{Data extraction and analysis}

For each survey included in the review the following items were extracted by one author (JR or WZ): the location, the year the survey concluded, the year it was published, and whether the RAAB protocol was used. We also recorded whether it was published in a scientific journal, and whether the journal was open-access on 1 December 2016. For RAABs, we recorded whether it was registered on the Repository, and whether a survey report, summary tables of results and the survey dataset had been uploaded to the Repository. 
Surveys were categorised as those

i) only published in a scientific journal;

ii) only with RAAB results tables on the Repository;

iii) published and with RAAB results tables on the Repository; and

iv) only registered on the Repository.

Descriptive analysis included the proportion of surveys that used the RAAB protocol; the proportion that were published and the lag-time to publishing from the time of survey completion; the proportion making tables and datasets available; and the proportion published in an open-access journal.

Users of survey results may require different blindness information, so reports with a wider range of information (i.e. more 'complete') are arguably more useful. To assess completeness of information within surveys we extracted data on whether surveys reported blindness prevalence by one or both of:

i) best-corrected and presenting visual acuity;

ii) a cut-off of $<3 / 60$ and $<6 / 60$, the World Health Organization categories of blindness and severe visual impairment;

iii) unilateral and bilateral;

iv) the sample prevalence and the prevalence adjusted to the age and sex profile of the survey area;

v) overall as well as disaggregated by sex.

Finally, as a test of rigour, for the surveys that had two sources of information available (i.e. publication or survey report and RAAB tables) any discrepancy between the data reported in the two sources in relation to i) the number of participants, and ii) the sample blindness prevalence and confidence interval were noted.

Ethics exemption was granted for this literature review by from the London School of Hygiene \& Tropical Medicine (Reference 10779).

\section{RESULTS}




\section{Study selection}

A summary of study selection is presented in Figure 1. From the literature search a total of 4,818 publications were identified for screening, including 24 identified from citation lists of screened publications. Following screening of titles and abstracts, 253 publications were identified for full review; the majority were in English, with 44 in Chinese, 9 in Spanish and none identified in other languages. After the initial assessment against the inclusion and exclusion criteria, 109 records were excluded for reasons provided in Figure 1. This left 144 publications-including 15 in Chinese and three in Spanish—reporting 156 separate studies for inclusion in this analysis.

In addition to the literature search, the 266 RAAB surveys listed on the Repository on December 1 2016 were assessed against the inclusion and exclusion criteria. Of these, 76 were excluded for reasons provided in Figure 1, leaving 190 studies for inclusion in this analysis. Once duplicates appearing in both searches were removed, 279 separate studies were included in this analysis (listed in Supplemental Tables 1 and 2).

\section{Description of included studies}

The 279 surveys were undertaken in 68 countries (Table 1 ) and two-thirds used either RAAB or RACSS protocol ( $n=186 ; 67 \%)$. Countries with the most surveys were China (48), Vietnam (28), India (21), Bangladesh (14), Nepal (14) and Nigeria (14). One-quarter of LMICs with a population $\geq 20$ million (11/43) and $62 \%$ of LMICs with a population $<20$ million (58/93) had no survey undertaken between 2000 and 2014. Twenty-six countries had at least one survey that had sampled the whole country, but more commonly surveys were limited to regions within countries (Table 1).

\section{Published studies}

We identified 203 published surveys: 165 in a scientific journal (in 153 manuscripts, including 72 RAABs reported in 60 manuscripts) and 38 in a report of survey methods and results available on the Repository (Table 2).

Information on when a survey was completed was available for 188 of the published studies (93\%). The time to publication ranged from $<1$ year (for study reports) to 11 years, with a mean and standard deviation of $2.8 \pm 1.8$ years. Approximately half of the surveys were published within two years ( $n=99 ; 49 \%)$ of study completion, and $85 \%$ were published within five years of completion 
$(n=172)$. The number of surveys published increased over time (from 10 in 2000-4 to 70 in 2010-14), as did the proportion published in open-access journals (from 40\% in 2000-4 to 57\% in 2010-14; Table 2).

\section{RAABS}

Of the 186 RAABs, more than half $(n=110 ; 59 \%)$ had results published in scientific journals $(n=72)$ or in online reports $(n=38)$. Fifty RAABs $(27 \%)$ had datasets available-34 of these were published and 16 unpublished. Of the 76 unpublished RAABs (41\%) twenty-three had summary results tables and/or datasets available and 53 ( $28 \%$ of all identified RAABs) had no information available beyond registration on the Repository.

Of the 53 RAABS where no information was available, 16 (30\%) were conducted in the 3 years preceding the search, 11 (21\%) 4-5 years ago, and 26 (49\%) more than 5 years ago. Surveys without results available were most often conducted in Malaysia $(n=5)$, Indonesia $(n=5)$ and Vietnam $(n=4)$ (Supplemental Table 2).

\section{Available information within studies}

Table 3 shows reporting from the 226 surveys that were published and/or had RAAB tables available. Surveys with both RAAB tables and a study report available provided results for all five combinations of blindness definitions, while reporting was more variable for surveys that were published only (i.e. without summary results tables). Blindness prevalence was most commonly reported disaggregated by sex as well as overall (91\%). Reporting both $<3 / 60$ and $<6 / 60$ cut-offs was also frequent (69\%), followed by reporting both bilateral and unilateral blindness (67\%), the sample and adjusted prevalence (60\%) and finally, reporting best-corrected as well as presenting visual acuity (55\%). The assessment for rigour showed discrepancies between the tables and publication for both outcomes - the number of participants and blindness prevalence differed $14 \%$ and $15 \%$ of the time respectively.

\section{DISCUSSION}

To avoid waste, data from surveys should be summarised in a report which is made publicly available in a timely fashion, so that the results can be used by stakeholders for prioritising and 
planning future services. This review of blindness prevalence surveys undertaken between 2000 and 2014 identified some good practices in relation to availability of information. For surveys that were published, the majority achieved this within a reasonable time frame, and the proportion of surveys available from open-access journals has increased (Table 2). The Repository provided an accessible infrastructure to track publication of registered surveys, as well as housing survey results, reports and datasets. In an environment of reduced likelihood of publication in peer-reviewed journals, ${ }^{6}$ publication through the Repository itself may become an alternative. The Repository also increased availability of study information and improved completeness of reporting through the provision of RAAB results tables (Table 3 ) which contain more tables and graphs than can fit in a standard journal article.

This review also highlights where improvements can be made. First, survey reports remained unavailable for 1 in 5 of all identified RAABs [n=37] four or more years after survey completion. These surveys may yet be published in a scientific journal, but summary reports (and datasets) could be made available regardless, as demonstrated by the 23 unpublished studies with results tables available on the Repository. Failing to make a study report available is a waste of resources, and researchers must be accountable for the investment made by funding agencies, often using public resources. Unpublished research also has opportunity costs, including other research projects that could have been supported, and the services that members of the survey team-often eye health workers—could have delivered during time spent undertaking the survey.

Second, we identified discrepancies in the range of blindness information reported (Table 3). Beyond sharing of standardised results tables, the utility of survey findings would increase if comparable definitions were more consistently reported. Our findings reinforce the call for specific reporting guidelines to be developed for blindness prevalence surveys to ensure comparable information is collected and reported. ${ }^{18}$

Third, the global coverage of surveys is unequal, with some countries having a high number of surveys relative to their population, while other countries have no information available from surveys (Table 1). A better distribution could be achieved if guidance is developed on how often a population based survey of eye health / visual impairment is required for what population size. 
Fourth, only 1 in 6 surveys (16\%) provided full datasets. Whether researchers want to share data or not, the era of data sharing in epidemiology has arrived ${ }^{19}$ and is increasingly expected. Ophthalmic epidemiology has already seen the benefits of data sharing in syntheses to estimate global blindness $^{9,12}$ and the incidence of vision-impairing cataract. ${ }^{20,21}$ Another benefit of data sharing is the improvement in data quality that occurs when it is known it will ultimately be shared. ${ }^{22}$ The discrepancies we identified between the datasets and published reports (Table 3) indicate the need for quality improvement in reporting of information. Beyond ensuring appropriate consent processes are in place, questions remain for implementation of data sharing, such as how to assure the quality of analyses arising from data sharing, and how to manage derived variables. ${ }^{19}$ The societal advantages of data sharing require stakeholders to address these questions rather than use them as an excuse for inaction, and fortunately guidance is emerging. ${ }^{22,23}$

Our analysis must be interpreted in the context of its limitations. We were unable to assess nonRAAB surveys that have been completed yet remain unpublished, as there is currently no registration process or repository for these studies. Further, registration on the Repository is currently not compulsory for RAABs, so it is likely that there are completed RAABs which we did not identify. For example, non-government organisations may have funded surveys that remain unregistered and unpublished. These missing studies can be reduced in future by enhancing the registration role of the Repository, perhaps through compulsory prospective registration e.g. registration as a requirement for use of the RAAB survey tools, or receiving funding.

We were also unable to assess whether surveys had been published locally and used for local planning, as no mechanism currently exists to measure this. It is possible some of the surveys we categorised as unpublished have been summarised in reports not identified by our search. This information gap could be overcome in future if principal investigators reported to the Repository how survey results were disseminated locally. While we performed our search without language restriction, it is likely that the databases we searched fail to index every survey published in a language other than English.22,24 Finally, we did not contact researchers listed on the Repository to request the tables and datasets from their study or to find whether reports were available elsewhere. Doing so may have increased the proportion of surveys for which information was available, but our results represent what is readily available and accessible. 
Beyond increasing the availability of information, strategies are also required to increase the translation of research to policy and planning. Failing to analyse the results of prevalence surveys within the local context to inform planning and action is possibly the biggest (if currently unquantified) source of waste in ophthalmic epidemiology. Together with spending on prevalence surveys it is important that resources are also made available to translate the evidence into meaningful activities based on the results of the survey, and to do more intervention research to identify effective strategies to reduce blindness. ${ }^{25}$

Investment in prevalence surveys in LMICs is set to continue in the pursuit of universal eye health. ${ }^{2}$ Availability and quality of information from blindness prevalence surveys would improve if prospective survey registration and access to study reports and datasets becomes the expectation rather than the exception. ${ }^{26}$ The RAAB Repository facilitates survey registration, full and consistent reporting of results and universal data access and in so doing provides a rare and valuable infrastructure 27,28 to disseminate survey information. We hope that this analysis promotes discussion amongst the ophthalmic community, increases the availability of study information, reduces avoidable waste from surveys and ultimately improves eye health. 


\section{REFERENCES}

1. World Health Assembly. Towards universal eye health: A global action plan 2014-2019. Resolution 66.4, 24 May 2013. Geneva: World Health Assembly, 2013.

2. World Health Organization. Universal Eye Health: A global action plan 2014-2019. Geneva: World Health Organization, 2013.

3. International Centre for Eye Health. Rapid Assessment of Avoidable Blindness. Secondary Rapid Assessment of Avoidable Blindness 2014. Available at: http://iceh.Ishtm.ac.uk/rapidassessment-of-avoidable-blindness/.

4. Dineen B, Foster A, Faal H. A proposed rapid methodology to assess the prevalence and causes of blindness and visual impairment. Ophthalmic Epidemiol. 2006;13(1):31-34.

5. Kuper H, Polack S, Limburg H. Rapid assessment of avoidable blindness. Community Eye Health. 2006;19(60):68-69.

6. West S. Passing the baton: reflections on publishing in Ophthalmic Epidemiology. Ophthalmic Epidemiol. 2011;18(4):143-145.

7. Health Information Services. RAAB repository. Secondary RAAB repository 2014. http://www.raabdata.info/.

8. Chalmers I, Glasziou P. Avoidable waste in the production and reporting of research evidence. Lancet. 2009;374:86-89.

9. Pascolini D, Mariotti SP. Global estimates of visual impairment: 2010. Br J Ophthalmol. 2012;96(5):614-618.

10. Pascolini D, Mariotti S, Pokharel G, et al. 2002 global update of available data on visual impairment: a compilation of population-based prevalence studies. Ophthalmic Epidemiol. 2004;11(2):67-115.

11. Bourne R, Price H, Taylor $\mathrm{H}$, et al. New Systematic Review Methodology for Visual Impairment and Blindness for the 2010 Global Burden of Disease Study. Ophthalmic Epidemiol. 2013;20(1):33-39.

12. Stevens GA, White RA, Flaxman SR, et al. Global prevalence of vision impairment and blindness: Magnitude and temporal trends, 1990-2010. Ophthalmology. 2013;120(12):23772384.

13. Abou-Gareeb I, Lewallen S, Bassett K, et al. Gender and blindness: a meta-analysis of population-based prevalence surveys. Ophthalmic Epidemiol. 2001;8(1):39-56.

14. Furtado JM, Lansingh VC, Carter MJ, et al. Causes of Blindness and Visual Impairment in Latin America. Surv Ophthalmol. 2011;57(2):149-177.

15. Murthy GV, Johnson GJ. Prevalence, incidence and distribution of visual impairment. In: Johnson GJ, Minassian DC, Weale RA, et al., eds. The epidemiology of eye disease. Third ed. London: Imperial College Press, 2012.

16. Munoz B, West S. Blindness and visual impairment in the Americas and the Caribbean. $\mathrm{Br} \mathrm{J}$ Ophthalmol. 2002;86(5):498-504.

17. World Bank. Countries and economies. Secondary Countries and economies 2014. Available at: http://data.worldbank.org/country.

18. Ramke J, Palagyi A, Jordan $\mathrm{V}$, et al. Using the STROBE statement to assess reporting in blindness prevalence surveys in low and middle income countries. PLoS One. In press.

19. Samet JM. Data: to share or not to share? Epidemiology. 2009;20(2):172-174.

20. Lewallen $S$, Courtright $P$, Etya'ale $D$, et al. Cataract incidence in sub-Saharan Africa: What does mathematical modeling tell us about geographic variations and surgical needs? Ophthalmic Epidemiol. 2013;20(5):260-266.

21. Lewallen S, Perez-Straziota $C$, Lansingh $V$, et al. Variation in cataract surgery needs in Latin America. Arch Ophthalmol. 2012;130(12):1575-1578.

22. Chan A-W, Song F, Vickers A, et al. Increasing value and reducing waste: addressing inaccessible research. Lancet. 2014;383:257-266.

23. Hrynaszkiewicz I, Norton ML, Vickers AJ, et al. Preparing raw clinical data for publication: guidance for journal editors, authors, and peer reviewers. Trials. 2010;11(9).

24. Xia J, Wright J, Adams CE. Five large Chinese biomedical bibliographic databases: accessibility and coverage. Health Info Libr J. 2008;25(1):55-61.

25. Ramke J, Zwi AB, Palagyi A, et al. Equity and blindness: closing evidence gaps to support universal eye health. Ophthalmic Epidemiol. 2015;22(5):297-307.

26. Erren T, Shaw D, Groß J. How to avoid haste and waste in occupational, environmental and public health research. J Epidemiol Community Health. 2015:jech-2015-205543.

27. Poole C. A vision of accessible epidemiology. Epidemiology. 2010;21(5):616-618.

28. Lash TL, Vandenbroucke JP. Should Preregistration of Epidemiologic Study Protocols Become Compulsory? Reflections and a Counterproposal. Epidemiology. 2012;23(2):184-188. 
Table 1: Blindness prevalence surveys undertaken in low and middle income countries 2000-2014

\begin{tabular}{|c|c|c|c|c|c|}
\hline \multirow{2}{*}{ Country } & \multirow{2}{*}{$\begin{array}{c}\text { World bank estimated } \\
2010 \text { population } \\
\text { (millions)* }^{*}\end{array}$} & \multicolumn{3}{|c|}{ Time period } & \multirow{2}{*}{ Total } \\
\hline & & $2000-4$ & $2005-9$ & 2010-14 & \\
\hline \multicolumn{6}{|l|}{ Countries <20 million } \\
\hline Bhutan† & 0.7 & - & 1 & - & 1 \\
\hline Bolivia† & 9.9 & - & - & 1 & 1 \\
\hline Burkina Fasso & 15.6 & - & - & 1 & 1 \\
\hline Burundi & 9.5 & - & - & 1 & 1 \\
\hline Cambodia & 14.4 & 3 & 1 & 1 & 5 \\
\hline Cuba & 11.3 & - & 1 & 1 & 2 \\
\hline Dominican Republic $†$ & 9.9 & - & 1 & - & 1 \\
\hline Ecuador† & 14.9 & - & 1 & - & 1 \\
\hline El Salvador† & 6.0 & - & - & 1 & 1 \\
\hline Eritrea† & 4.7 & - & 1 & - & 1 \\
\hline Fiji & 0.9 & - & 1 & - & 1 \\
\hline Guatemala & 14.7 & 1 & - & - & 1 \\
\hline Guinea Bissau† & 1.6 & - & - & 1 & 1 \\
\hline Honduras† & 7.5 & - & - & 1 & 1 \\
\hline Jordan & 6.0 & - & - & 1 & 1 \\
\hline Laos† & 6.3 & - & 1 & - & 1 \\
\hline Liberia† & 4.0 & - & - & 1 & 1 \\
\hline Libya† & 6.3 & - & - & 1 & 1 \\
\hline Malawi & 14.8 & - & 1 & 1 & 2 \\
\hline Mali & 15.2 & - & 1 & 1 & 2 \\
\hline Moldova† & 3.6 & - & - & 1 & 1 \\
\hline Mongolia† & 2.7 & - & - & 1 & 1 \\
\hline Occupied Palestinian Territories & 3.8 & - & 1 & - & 1 \\
\hline Panama & 3.6 & - & - & 1 & 1 \\
\hline Paraguay $\dagger$ & 6.2 & - & 1 & 1 & 2 \\
\hline Papua New Guinea & 6.8 & - & 1 & - & 1 \\
\hline Rwanda & 10.3 & - & 1 & - & 1 \\
\hline Senegal & 13.0 & - & - & 2 & 2 \\
\hline Sierra Leone† & 5.8 & - & - & 1 & 1 \\
\hline South Sudan & 10.1 & - & 1 & - & 1 \\
\hline Suriname $\dagger$ & 0.5 & - & - & 1 & 1 \\
\hline The Gambia† & 1.7 & - & 1 & - & 1 \\
\hline Timor-Leste† & 1.1 & - & 1 & 1 & 2 \\
\hline Turkmenistan† & 5.0 & 1 & - & - & 1 \\
\hline Zambia & 13.9 & - & - & 1 & 1 \\
\hline \multicolumn{6}{|l|}{ Countries $\geq 20$ million^ } \\
\hline Afghanistan & 28.0 & - & - & 3 & 3 \\
\hline Bangladesh†† & 151.6 & 1 & 4 & 9 & 14 \\
\hline Brazil & 198.6 & 2 & 1 & - & 3 \\
\hline Burma & 51.7 & 7 & 1 & 1 & 9 \\
\hline China & 1337.7 & 7 & 22 & 17 & 46 \\
\hline Colombia & 45.9 & - & 1 & - & 1 \\
\hline Egypt & 82.0 & - & 2 & - & 2 \\
\hline Ethiopia†† & 87.6 & 1 & 3 & 1 & 5 \\
\hline Ghana & 24.3 & 1 & 2 & - & 3 \\
\hline India & 1231.0 & 4 & 5 & 7 & 16 \\
\hline Indonesia & 241.6 & 3 & 1 & 3 & 7 \\
\hline Iran & 74.3 & 1 & 4 & 3 & 8 \\
\hline Kenya & 40.3 & 1 & 3 & 1 & 5 \\
\hline Madagascar & 21.1 & - & - & 1 & 1 \\
\hline Malaysia & 28.1 & 1 & - & 6 & 7 \\
\hline Mexico & 118.6 & - & 1 & 2 & 3 \\
\hline Mozambique & 24.3 & - & - & 2 & 2 \\
\hline Nepal & 26.9 & 1 & 11 & 2 & 14 \\
\hline Nigeria†† & 159.4 & 5 & 6 & 1 & 12 \\
\hline North Korea & 24.5 & - & - & 1 & 1 \\
\hline Pakistan†† & 170.0 & 2 & - & - & 2 \\
\hline Peru† & 29.4 & 1 & - & 1 & 2 \\
\hline Philippines & 93.0 & - & 2 & 1 & 3 \\
\hline South Africa & 50.8 & - & 2 & 2 & 4 \\
\hline Sri Lanka & 20.7 & - & 1 & - & 2 \\
\hline Sudan & 36.1 & - & - & 7 & 7 \\
\hline Tanzania & 45.6 & - & 2 & - & 2 \\
\hline Thailand $†$ & 66.7 & - & - & 1 & 1 \\
\hline Uganda & 33.1 & - & 1 & 3 & 3 \\
\hline Vietnam & 86.9 & 8 & 16 & 4 & 28 \\
\hline Yemen & 23.6 & 1 & 1 & 1 & 3 \\
\hline Total & & 52 & 109 & 103 & $264^{\wedge \wedge}$ \\
\hline
\end{tabular}

*downloaded from http://data.worldbank.org/indicator/SP.POP.TOTL [last updated 14/10/2015];

$\wedge 32 / 43(74 \%)$ LMICs with a population $\geq 20$ million in 2010 had at least one survey undertaken between 2000 and 2014 ; the 11 countries $\geq 20$ million population without at least one survey were Algeria, Angola, Congo, Cote d'Ivoire, Iraq, Morocco, Romania, Syrian Arab Republic, Turkey, Ukraine, Uzbekistan;

M15 studies did not report the date of the survey and were not included in this table (India=5; Cameroon, China, Nigeria=2; Botswana, Kenya, Pakistan, Tanzania=1). (Botswana study sampled from the national population);

$\dagger$ Includes at least one RAAB study that used a national sampling frame:

††Includes at least one non-RAAB study that used a national sampling frame. 
Table 2: Summary of included studies (blindness prevalence surveys in low and middle income countries 2000-2014)

\begin{tabular}{|c|c|c|c|}
\hline Description & RAAB studies* & $\begin{array}{c}\text { Non- RAAB } \\
\text { studies }\end{array}$ & All studies \\
\hline Included Studies & $n=186$ & $n=93$ & $n=279$ \\
\hline Number of countries & 63 & 25 & 68 \\
\hline \multicolumn{4}{|l|}{ Publication } \\
\hline Scientific journal & $\begin{array}{c}72 \text { (39\%) } \\
\text { (in } 60 \text { manuscripts) }\end{array}$ & $93(100 \%)$ & $165(59 \%)$ \\
\hline $\begin{array}{l}\text { Online report } \\
\text { Not published }\end{array}$ & $\begin{array}{l}38(20 \%) \\
76(41 \%)\end{array}$ & Unknown & $\begin{array}{l}38(14 \%) \\
76(27 \%)\end{array}$ \\
\hline \multicolumn{4}{|l|}{ Results Dissemination } \\
\hline \multicolumn{4}{|l|}{ On Repository } \\
\hline $\begin{array}{l}\text { Published, datasets and results } \\
\text { tables }\end{array}$ & $34(18 \%)$ & - & $34(12 \%)$ \\
\hline Published and results tables & $23(12 \%)$ & - & $23(8 \%)$ \\
\hline Datasets and results tables & $16(9 \%)$ & - & $16(6 \%)$ \\
\hline Results tables & $7(4 \%)$ & - & $7(3 \%)$ \\
\hline Registered only & $53(28 \%)$ & - & $53(19 \%)$ \\
\hline Published studies & $n=110$ & $n=93$ & $n=203$ \\
\hline \multicolumn{4}{|l|}{ Time to publication } \\
\hline Unknown** & $6(5 \%)$ & $9(10 \%)$ & $15(7 \%)$ \\
\hline$\leq 2$ years & $61(55 \%)$ & $38(41 \%)$ & $99(49 \%)$ \\
\hline$>2$ years $\leq 5$ years & $33(30 \%)$ & $40(43 \%)$ & $73(36 \%)$ \\
\hline$>5$ years & $10(9 \%)$ & $6(6 \%)$ & $16(8 \%)$ \\
\hline Scientific Journals & $n=60$ & $n=93$ & $n=153$ \\
\hline \multicolumn{4}{|l|}{ Language } \\
\hline English & $54(90 \%)$ & $78(84 \%)$ & $132(86 \%)$ \\
\hline Chinese & - & $15(16 \%)$ & $15(10 \%)$ \\
\hline Spanish & $6(10 \%)$ & - & $6(4 \%)$ \\
\hline \multicolumn{4}{|l|}{ Open-access (year published) $†$} \\
\hline $2000-4$ & $0 / 2(0 \%)$ & $4 / 8(50 \%)$ & $4 / 10(40 \%)$ \\
\hline $2005-9$ & 9/21 (43\%) & 19/35 (54\%) & $28 / 56(50 \%)$ \\
\hline 2010-14 & $20 / 33(61 \%)$ & $20 / 37(54 \%)$ & $40 / 70(57 \%)$ \\
\hline
\end{tabular}

* includes RACSS studies;

**study completion date not given;

†28 studies published after 2014 not included. 
Table 3: Availability of information within included studies* (blindness prevalence surveys in low and middle income countries 2000-2014)

\begin{tabular}{|c|c|c|c|c|}
\hline & \multicolumn{3}{|c|}{ Availability of study information } & \multirow{4}{*}{$\begin{array}{l}\text { Total } \\
n=226^{\wedge}\end{array}$} \\
\hline & \multicolumn{2}{|c|}{ Published Only } & \multirow{3}{*}{$\begin{array}{c}\text { Repository } \\
\text { RAAB Tables } \\
\pm \text { (Published }^{\text {Por Datasets) }}{ }^{* *} \\
\quad n=80\end{array}$} & \\
\hline & RAAB & $\begin{array}{l}\text { Not } \\
\text { RAAB }\end{array}$ & & \\
\hline & $n=53$ & $n=93$ & & \\
\hline Blindness definition & $\%$ & $\%$ & $\%$ & $\%$ \\
\hline \multicolumn{5}{|l|}{$\begin{array}{l}\text { Proportion of studies that report blindness } \\
\text { in terms of both: }\end{array}$} \\
\hline Best-corrected and presenting visual acuity & 26 & 33 & 100 & 55 \\
\hline$<3 / 60$ and $<6 / 60$ cut-off & 75 & 39 & 100 & 69 \\
\hline Unilateral and bilateral visual acuity & 47 & 51 & 100 & 67 \\
\hline Sample and adjusted & 60 & 26 & 100 & 60 \\
\hline Overall and disaggregated by sex & 83 & 87 & 100 & 91 \\
\hline Rigour & & & $\%(n)$ & \\
\hline \multicolumn{5}{|l|}{$\begin{array}{l}\text { Proportion of studies with consistent } \\
\text { results reported in RAAB tables and } \\
\text { published report }\end{array}$} \\
\hline Number of participants & - & - & $86(49 / 57)$ & - \\
\hline Blindness prevalence $\dagger$ & - & - & $85(47 / 55)$ & - \\
\hline
\end{tabular}

*53 studies registered on the RAAB Repository with no further information were not included in this table; ** includes 57 studies that were published;

† 2 studies did not report sample prevalence in the publication so this item could not be assessed. 